\title{
SIMULADOR DE PACIENTE T1D EN TIEMPO REAL
}

\author{
Juliana Manrique Cordoba \\ Universidad del Cauca, Popayán - Colombia, carlmanri@unicauca.edu.co \\ Juan David Romero Ante \\ Universidad del Cauca, Popayán - Colombia, rdavid@unicauca.edu.co \\ José María Sabater Navarro \\ Universidad Miguel Hernández, Elche - España, j.sabater@goumh.umh.es \\ Oscar Andrés Vivas Albán \\ Universidad del Cauca, Popayán - Colombia, avivas@unicauca.edu.co \\ José María Vicente Samper \\ Universidad Miguel Hernández, Elche - España, jose.vicentes@umh.es
}

\section{Resumen}

Este artículo desarrolla una implementación en tiempo real de un modelo matemático que describe la dinámica glucosa - insulina de un paciente con Diabetes Mellitus Tipo 1 (T1D). Adicionalmente, se realizan aportes al modelo para contemplar la ingesta de grasas y proteinas, de manera que se pueda evidenciar la influencia de estas sobre el nivel de glucosa en sangre. También, se considera la infusión de insulina exógena a través de MDI o ISCI, teniendo en cuenta diferentes tipos de insulina, y la variación de la sensibilidad a la insulina durante el día. Asimismo, se muestra el acople del modelo a una herramienta de monitorización remota que actualmente se utiliza en casos reales. La implementación en tiempo real permite demostrar que desde la ingeniería se pueden realizar aportes al tratamiento de la T1D.

Palabras clave: Modelo matemático, glucosa insulina, T1D, tiempo real.

\section{INTRODUCCIÓN}

Diferentes modelos matemáticos del sistema glucosa - insulina se han propuesto desde finales de la década de 1970 [6, 12, 19], estos modelos se han usado para realizar una cantidad significativa de estudios de simulación. Conocer el comportamiento de las variables involucradas en el proceso demuestra la necesidad de implementar controles cerrados para la terapia insulínica [7].

La mayoría de los modelos existentes en el área, son dedicados a las dinámicas de datos de prueba de tolerancia a la glucosa intravenosa (IVGTT), prueba de glucosa oral (OGTT) y prueba de tolerancia a la glucosa intravenosa muestreada con frecuencia (FSIGT) [5]. Una de las aproximaciones de los modelos matemáticos de la dinámica glucosa insulina está basada en el concepto de compartimiento. Un compartimiento representa la reserva de una sustancia y el flujo de intercambio de masa [18]; los compartimientos que suelen definirse son la glucosa y la insulina en plasma.

Los procesos fisiológicos que intervienen en el ser humano son muy complejos, impidiendo conseguir un modelo que describa de forma exacta la dinámica glucosa - insulina. En 1980, Bergman y Cobelli [4] proponen un modelo que contiene un número mínimo de parámetros y se usa en trabajos de investigación fisiológica para estimar los efectos de la glucosa y la sensibilidad a la insulina; en 1989, Berger y Rodbard [3] desarrollan otro modelo que tiene en cuenta la ingesta de carbohidratos, la administración de insulina exógena y describen la cinemática de diferentes tipos de insulina, basados en una ecuación logística de absorción de insulina; posteriormente en 1992, Lehmann y Deutsch [13] se basan en el modelo de Berger para mejorar la simulación y reflejar la fisiología de la acción de la insulina y la absorción de carbohidratos en términos cuantitativos; Fabietti [10], lleva a cabo un modelo de la dinámica glucosa insulina específicamente creado para facilitar el diseño y evaluación de algoritmos de control que se desean implementar en lazo cerrado, el modelo de Fabietti se basa en el propuesto por Bergman y Cobelli.

El modelo matemático desarrollado por Dalla Man et al. [9] publicado en el año 2007, consta de una serie de ecuaciones diferenciales ordinarias, que permiten describir el comportamiento de la dinámica glucosa insulina en pacientes sanos y con Diabetes Mellitus 
Tipo 2 (T2D); este es un modelo de compartimientos en el que se divide el organismo en subsistemas que detallan la producción y utilización de glucosa e insulina por parte del hígado, los riñones y el páncreas.

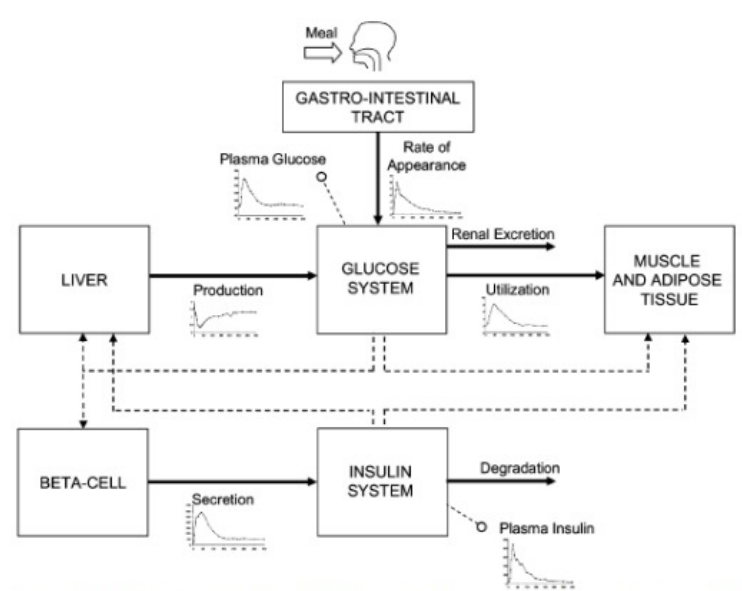

Figura 1: Esquema de dinámica glucosa - insulina desarrollado por Dalla Man et al. [9]

Disponer de un modelo matemático que permita simular un paciente con Diabetes Mellitus Tipo 1 (T1D), facilita la educación sobre los factores que pueden afectar los niveles de azúcar en la sangre, teniendo en cuenta que la T1D es una enfermedad que requiere tratamiento vitalicio, generalmente administrado por el propio paciente. Además, es relevante como herramienta docente para el estudio de la enfermedad [17], permite la realización de pruebas de estrategias de control, en la regulación del nivel de glucosa, favorece el estudio de manera cuantitativa sin el riesgo de experimentar directamente en el paciente; de igual manera, impulsa el desarrollo de algoritmos de predicción basados en el modelo matemático que proporcionen un control óptimo del nivel de glucosa en sangre [18].

\section{MODELO MATEMÁTICO}

Se propone involucrar en el modelo matemático de la dinámica glucosa - insulina para pacientes con T1D, información relacionada con la ingesta de carbohidratos, grasas y proteínas; la infusión de diferentes tipos de insulina exógena, tanto por Múltiples Dosis de Insulina (MDI) como por Infusión Subcutánea Continua de Insulina (ISCI); además de tener en cuenta los efectos de la saturación de la insulina en la zona de inserción, para el caso de los tratamientos que implementan dispositivos ISCI y la variación de la utilización de glucosa a diferentes horas del día.

A continuación se desarrolla la implementación de las ecuaciones y modelos utilizados para reproducir la dinámica del organismo, desde la ingesta de alimentos hasta la aparición de glucosa en el torrente sanguíneo. La Figura 2 ilustra el diagrama de bloques que representa los factores que afectan la dinámica glucosa - insulina en el cuerpo; el núcleo del sistema está representado con la línea punteada; la interacción entre la glucosa y la insulina se diseñó modificando algunos de los parámetros establecidos por Dalla Man et al. [9] para pacientes sanos, teniendo en cuenta que la T1D se caracteriza por tener una deficiencia en la producción de insulina, a diferencia de la T2D en la que el cuerpo no hace uso apropiado de la misma [1].

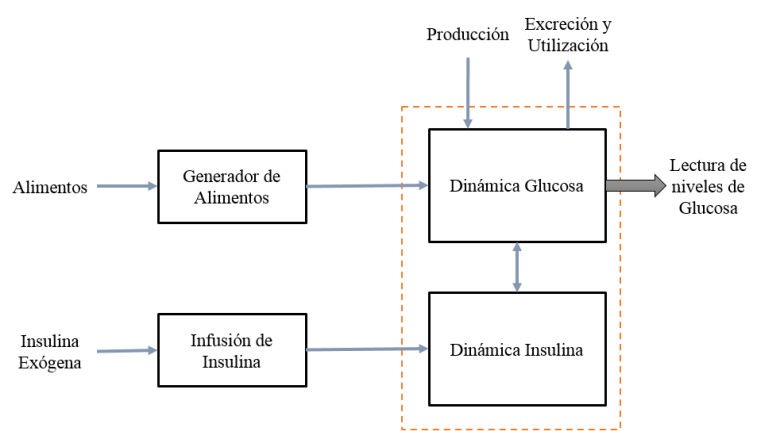

Figura 2: Diagrama de bloques del modelo matemático de paciente con T1D.

\subsection{MODELO DE DINÁMICA GLUCOSA - INSULINA}

El modelo se compone de dos subsistemas principales que describen la evolución de la glucosa y la insulina en plasma, asociados a subsistemas adicionales que modelan la producción y utilización de glucosa e insulina por parte de los riñones, el hígado y el páncreas. La cinemática de la glucosa se modela a partir de dos compartimientos, la cantidad de glucosa en plasma y en tejidos. Del mismo modo, el subsistema de la insulina se compone de la cantidad de insulina en el hígado y en el plasma sanguíneo. La cantidad de glucosa se debe a la ingesta directa de alimento, la producción endógena y la utilización de la misma. La tasa de aparición de glucosa es producto de la absorción intestinal, que describe el tránsito de la glucosa a través del estómago, representado por dos compartimientos para diferenciar las fases de los alimentos sólidos y triturados, y el intestino. La utilización total de glucosa es la suma entre la utilización independiente y dependiente de insulina, la primera hace referencia a la glucosa consumida por el cerebro y la segunda está relacionada con la cantidad de glucosa en tejidos y la cantidad de insulina que pasa por tejido intersticial.

\subsubsection{Adaptación del modelo para T1D}

Para representar la deficiencia en la producción de insulina de un paciente con T1D se asume que la 
secreción de insulina $(S(t))$ es nula en el subsistema BETA-CELL del modelo presentado en [9]; se modifican los valores correspondientes a los parámetros $\beta$ y $K$ que corresponden a la respuesta pancreática a la glucosa y al cambio de glucosa respectivamente; asimismo la secreción basal $\left(S_{b}\right)$, la insulina en plasma basal $\left(I_{p b}\right)$ y la insulina en el hígado basal $\left(I_{l b}\right)$ toman un valor de cero.
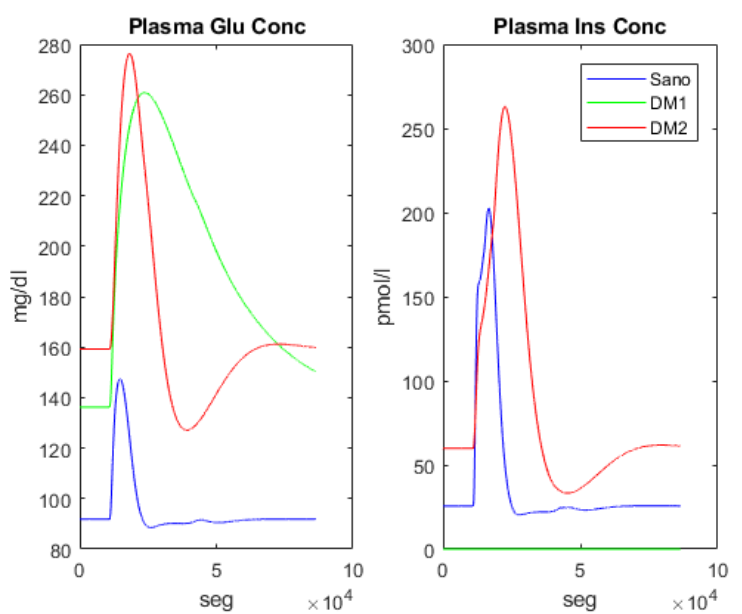

Figura 3: Respuesta de simulación con ingesta de 70 gramos de carbohidratos.

La Figura 3 ilustra la concentración de glucosa e insulina en plasma para cada tipo de paciente; en los pacientes sano y con T2D se simulan a partir del modelo presentado en [9], mientras que la respuesta del paciente con T1D se obtiene a partir de las modificaciones mencionadas previamente; es de destacar que la respuesta de la concentración de insulina en plasma es cero en el paciente con T1D.

\subsubsection{Adaptación del modelo para variación de sensibilidad a la insulina}

La sensibilidad a la insulina es la relación de la cantidad de insulina que el organismo necesita producir para contrarrestar cierta proporción de glucosa; sin embargo, esta puede ser perturbada por factores externos como el estrés, el ciclo menstrual en el caso de las mujeres, actividad física, enfermedades infecciosas, ingestas de alimentos que alteren el ritmo cardíaco o la producción de glucosa en el hígado, como el café y el alcohol, entre otros. En el modelo matemático se hace una adaptación para representar dichas perturbaciones con el parámetro $K_{m 0}$ del subsistema de utilización de glucosa, que de acuerdo a las ecuaciones $(1,2)$ es inversamente proporcional al consumo de glucosa dependiente de la insulina $\left(U_{i d}(t)\right)$ y por consiguiente directamente al nivel de glucosa.

$$
U_{i d}(t)=\frac{V_{m}(X(t)) \cdot G_{t}(t)}{K_{m}(X(t))+G_{t}(t)}
$$

$$
K_{m}(X(t))=K_{m 0}+K_{m x} X(t)
$$

Para la implementación en el modelo matemático se definen lapsos de tiempo $\left(T_{\text {sen }}\right)$ y sensibilidades que corresponden a cada uno de estos, de tal manera que $K_{m 0}$ varíe en el tiempo con respecto a la sensibilidad definida, lo anterior se realiza de acuerdo a un porcentaje de variación $\left(P_{v a r}\right)$ y a los valores máximos y mínimos de la sensibilidad.

$$
\begin{aligned}
& K_{m_{0}}(t)=\min K+\operatorname{var} \operatorname{Sen}(1)+
\end{aligned}
$$

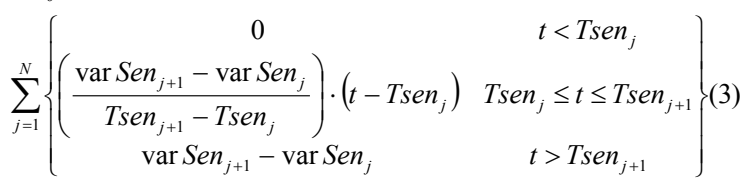

Se define varSen como la variación de la sensibilidad para cada instante de tiempo, la cual se obtiene a partir del porcentaje $P_{v a r}$ alrededor del valor de $K_{m} 0$ establecido en [9].

\subsection{GENERADOR DE ALIMENTOS}

El modelo de absorción de alimentos propuesto en [9] toma únicamente la ingesta de glucosa presente en los carbohidratos; sin embargo las grasas y proteínas aportan glucosa en diferente proporción y velocidad de metabolización. La Sociedad Española de Diabetes [20] define la Unidad Grasa - Proteína (UGP) como la cantidad de alimento que aportan 150 kilocalorías (kcal) de grasa y/o alimentos ricos en proteína, teniendo en cuenta que 1 gramo de grasa y 1 gramo de proteína corresponden a $9 \mathrm{kcal}$ y $4 \mathrm{kcal}$ respectivamente. Para metabolizar 1 UGP se necesitan 3 horas y por cada UGP adicional se añade 1 hora, es decir, si para 1 UGP se contabilizan 3 horas, para 2 UGP, 4 horas; para 3 UGP, 5 horas; para más de 4 UGP, de 6 a 8 horas [21].

El modelo de absorción oral de glucosa tiene como entrada la aparición instantánea de glucosa en el estómago proveniente de las ingestas $(N)$ de carbohidratos $(\operatorname{MealC}(t))$ y UGP $(\operatorname{MealGP}(t))$, a determinadas horas $(M)$; ahora bien, para modelar la metabolización de las UGP, se implementa una función lineal creciente en el tiempo de acuerdo a la duración de digestión $\left(t_{\text {dig }}\right)$ definida anteriormente.

$$
\begin{gathered}
\text { MealC }(t)=\sum_{j=1}^{N}\left\{\begin{array}{cc}
0 & t<M \\
C & t \geq M
\end{array}\right\} \\
\text { MealGP }(t)=\sum_{j=1}^{N}\left\{\begin{array}{cc}
0 & t<M_{j} \\
\left(\frac{U G P}{t_{\text {dig }}}\right)_{U G P}\left(t-M_{j}\right) & M_{j} \leq t \leq t_{\text {dig }}+M_{j} \\
t>t_{\text {dig }}+M_{j}
\end{array}\right\}(5)
\end{gathered}
$$


Dado que en la absorción oral se ingresa la glucosa proveniente de los carbohidratos se debe sumar lo correspondiente en glucosa de las UGP para obtener el consumo total de alimento; estas aportan el equivalente a 0.1 gramos de carbohidratos.

\subsection{MODELO DE INFUSIÓN SUBCUTÁNEA}

La tasa de absorción de insulina subcutánea en plasma $I_{\text {exo }}(t)$, se definió como la suma de las posibles administraciones de insulina subcutánea (MDI o ISCI) en el tiempo, así:

$$
I_{e x o}(t)=I_{a b s}(t)+K_{\text {inf }}(t) \cdot R_{i}(t)
$$

Siendo $I_{a b s}(t)$ y $R_{i}(t)$ la tasa de aparición de insulina en plasma por MDI o ISCI respectivamente en (pmol / $\mathrm{kg} /$ segundos); $K_{\text {inf }}(t)$ describe la saturación de insulina en la zona de inserción para el caso del uso de dispositivos ISCI, los cuales al ser utilizados por lapsos de tiempo prolongados pueden provocar infecciones, filtración de insulina y formación de burbujas [2]; provocando una disminución de la eficiencia en la absorción de la insulina. En el modelo matemático $K_{\text {inf }}(t)$ es una función decreciente en el tiempo:

$$
K_{\text {inf }}(t)=P_{k} \cdot t
$$

$P_{k}$ es el parámetro que representa el porcentaje de pérdida de eficiencia por día.

\subsubsection{Múltiples Dosis de Insulina (MDI)}

En el modelo por MDI se tiene en cuenta las cinemáticas de diferentes tipos de insulinas (Regular, $\mathrm{NPH}$, lenta y ultralenta) descritas en [3]. $I_{a b s}(t)$ se define en la ecuación (8), donde $D^{n}$ es la cantidad de Unidades de Insulina (UI) inyectada, $t$ es el tiempo después de la inyección, $s$ es el parámetro que define el patrón de absorción de insulina despendiendo del tipo. Cabe resaltar que las unidades de $I_{a b s}(t)$ definidas en [3] son UI / segundos y las unidades empleadas en el modelo son pmol / $\mathrm{kg} / \mathrm{segundos}$, se define el parámetro $z$ teniendo en cuenta el peso molecular de la insulina tipo Aspart (5831.648 gramos / mol)[20] para la adecuación de unidades.

$$
I_{a b s}(t)=\sum_{j=1}^{N} z \cdot \frac{s \cdot t^{s} \cdot T_{50}^{s} \cdot D_{j}^{n}}{t\left[T_{50}^{s}+t^{s}\right]^{2}}
$$

$T_{50}$ es el intervalo de tiempo para alcanzar una absorción del $50 \%$ de la insulina inyectada descrito en la ecuación (6).

$$
T_{50}\left(D^{n}\right)=a \cdot D^{n}+b
$$

Siendo a y b parámetros definidos por Berger y Rodbard para caracterizar la dependencia del intervalo de tiempo.

\subsubsection{Infusión Subcutánea Continúa de Insulina (ISCI)}

En el módulo ISCI se implementa una variación del modelo de la cinemática de insulina subcutánea presentado en [8], donde la infusión $(\operatorname{IIR}(t))$ se modela como un sistema de dos compartimientos, que representan las concentraciones subcutáneas de insulinas monomérica y no monomérica.

$$
\begin{aligned}
& \dot{I}_{s c 1}(t)=-\left(k_{d}+k_{a 1}\right) \cdot I_{s c 1}(t)+\operatorname{IIR}(t) \\
& \dot{I}_{s c 2}(t)=k_{d} \cdot I_{s c 1}(t)-k_{a 2} \cdot I_{s c 2}(t) \\
& R_{i}(t)=k_{a 1} \cdot I_{s c 1}(t)+k_{a 2} \cdot I_{s c 2}(t)
\end{aligned}
$$

Donde $I_{s c 2}(t)$ y $I_{s c 1}(t)$ son las cantidades de insulina monomérica y no monomérica respectivamente, $k_{d}$ es la tasa de descomposición de insulina inyectada, $k_{a l} \mathrm{y}$ $k_{a 2}$ son parámetros de transmisión de insulina $\mathrm{y}$ $\operatorname{IIR}(t)$ es la tasa de infusión de insulina, que a su vez se define como la suma de las tasas de insulina por bolo normal $\left(I I R_{n}\right)$, bolo cuadrado $\left(I I R_{c}\right)$ y basal $\left(I I R_{b}\right)$.

$$
\begin{gathered}
I I R(t)=z \cdot\left(I I R_{n}(t)+I I R_{c}(t)+I I R_{b}(t)\right) \\
I I R_{n}(t)=\sum_{j=1}^{N i n} D_{j}^{n} \cdot \delta\left(t-t_{j}^{n}\right) \\
I I R_{c}(t)=\sum_{j=1}^{N i c}\left\{\begin{array}{cc}
D_{j}^{c} & t_{j}^{n} \leq t<\left(t_{j}^{n}+L_{j}^{c}\right) \\
0 & t_{j}^{n}>t \geq\left(t_{j}^{n}+L_{j}^{c}\right)
\end{array}\right\} \\
I I R_{b}(t)=\sum_{j=1}^{N i b}\left\{\begin{array}{cc}
D_{j}^{b} & t_{j}^{b} \leq t<t_{j+1}^{b} \\
0 & t_{j}^{b}>t \geq t_{j+1}^{b}
\end{array}\right\}
\end{gathered}
$$

Siendo Nib, Nin, Nic el número de infusiones de insulina basal, por bolo normal y bolo cuadrado respectivamente; los superíndices $b, n$ y $c$ representan las infusiones de insulina basal, bolo normal y bolo cuadrado, $D$ es la dosis de insulina inyectada, $t$ el instante de tiempo en el que se realiza la infusión y $L$ es la duración del bolo cuadrado.

\subsection{IMPLEMENTACIÓN EN TIEMPO REAL}


La implementación en tiempo real del modelo de dinámica glucosa - insulina, simula un paciente real y permite evaluar el desempeño tanto de controladores como de dispositivos de infusión continua de insulina. Asimismo, la utilización de este tipo de implementación permite valorar el desempeño de herramientas de visualización remota. Para la ejecución del modelo en tiempo real fue necesario ajustar los parámetros que incluyeran unidades de tiempo, de minutos a segundos.

El modelo fue implementado en la herramienta Simulink de Matlab, ejecutado en el modo de simulación externo, Simulink cuenta con el paquete de soporte (Simulink Support Package for Raspberry $P i$ Hardware), que permite establecer la comunicación con el dispositivo hardware Raspberry $\mathrm{Pi}$, de manera que la simulación del modelo se ejecuta con el tiempo contabilizado por el reloj de la placa.

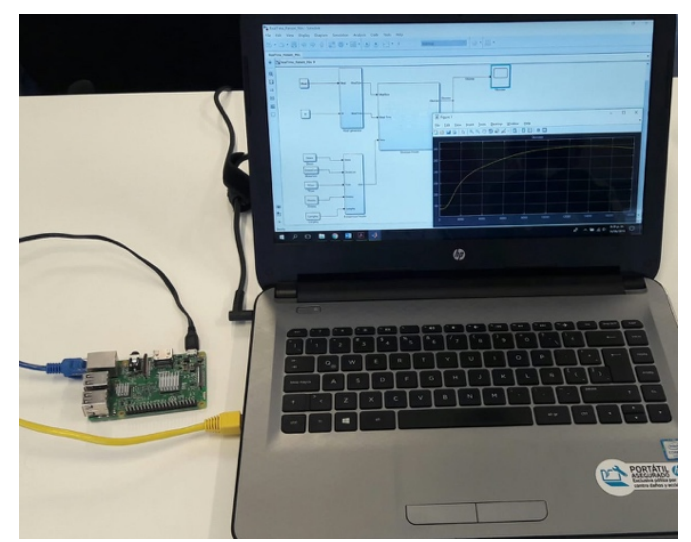

Figura 4: Fotografía de conexión entre Simulink y Raspberry Pi.

\subsection{MONITORIZACIÓN REMOTA}

La monitorización remota se establece utilizando la herramienta Nightscout [16], que a vez es un repositorio desarrollado para pacientes con diabetes, este hace posible la visualización en tiempo real del nivel de glucosa. Nightscout es una aplicación que se desarrolla sobre una plataforma de computación en la nube denominada Heroku [11], que permite el despliegue de aplicaciones y la creación de una base de datos en MongoDB [14].

Matlab permite establecer comunicación con la base de datos en MongoDB por medio de un paquete de soporte. Al igual que el funcionamiento de los Monitores Continuos de Glucosa (CGM), haciendo uso del paquete de comunicación, desde Matlab se envía el estado del nivel de glucosa, cada cinco minutos mientras se ejecuta la simulación, a la base de datos para su visualización en Nightscout.
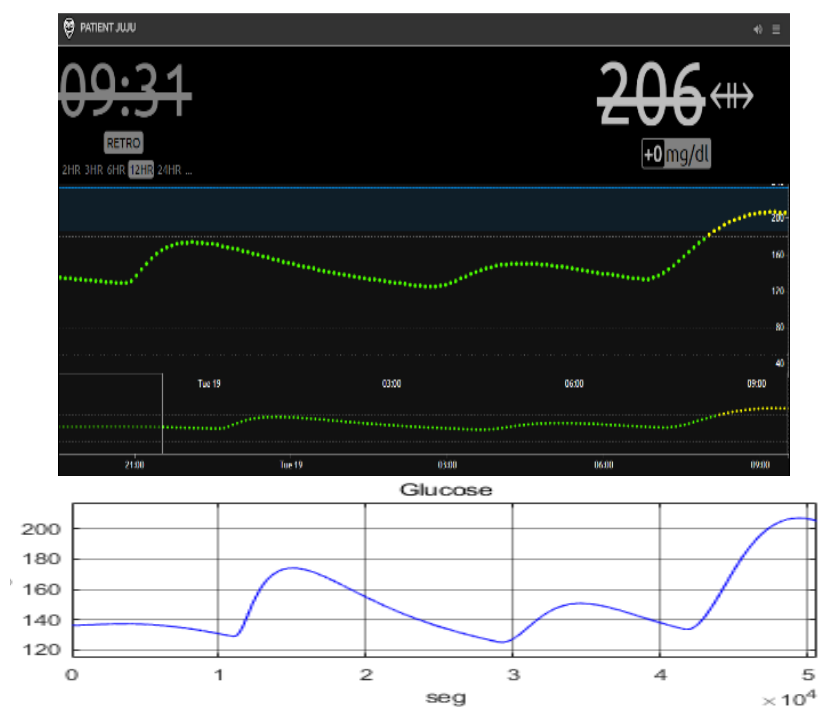

Figura 5: Visualización del nivel de glucosa en Nightscout y Simulink.

\section{RESULTADOS DE SIMULACIÓN}

En los resultados de simulación se aprecia los aportes realizados al modelo matemático, que fueron detallados en la sección anterior.

\subsection{INGESTA DE CARBOHIDRATOS, GRASAS Y PROTEÍNAS}

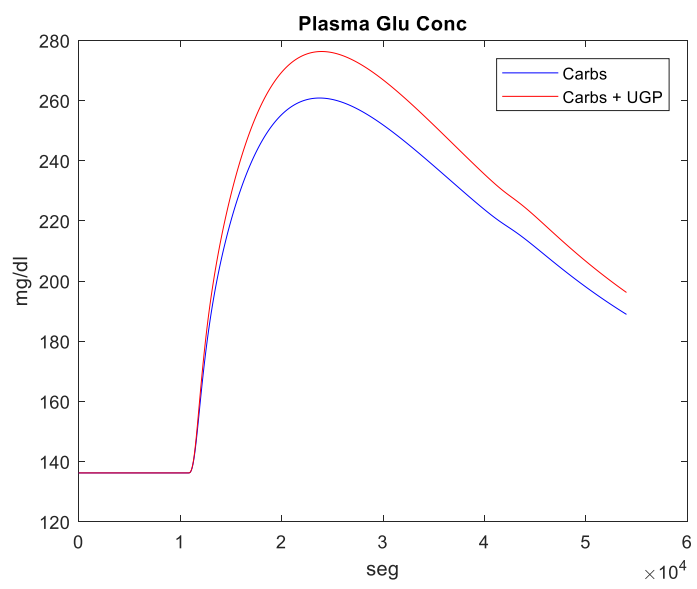

Figura 5: Simulación de ingesta de carbohidratos vs. carbohidratos, grasas y proteínas.

La Figura 5 ilustra el efecto adicional que tiene la ingesta de grasas y proteínas en un paciente con T1D. Se simula una ingesta única de 70 gramos de carbohidratos y una ingesta de igual proporción en carbohidratos con 10 gramos de grasas y proteínas adicionales. El aporte de glucosa en sangre de las grasas y proteínas no es representativo con respecto al aporte suministrado por los carbohidratos; sin embargo, deben ser considerados en el tratamiento por su contribución de azúcar a la sangre. 


\section{2}

\section{INFUSIÓN} INSULINA

SUBCUTÁNEA

DE

En la Figura 6 se aprecia la respuesta del modelo con los tipos de tratamiento, MDI e ISCI con igual cantidad de insulina en ambos casos; en esta simulación se puede evaluar las ventajas $\mathrm{y}$ desventajas que puede presentar cada tratamiento. Las dos simulaciones incluyeron una ingesta de 50 gramos de carbohidratos y 40 gramos de grasas y 40 graos de proteínas.
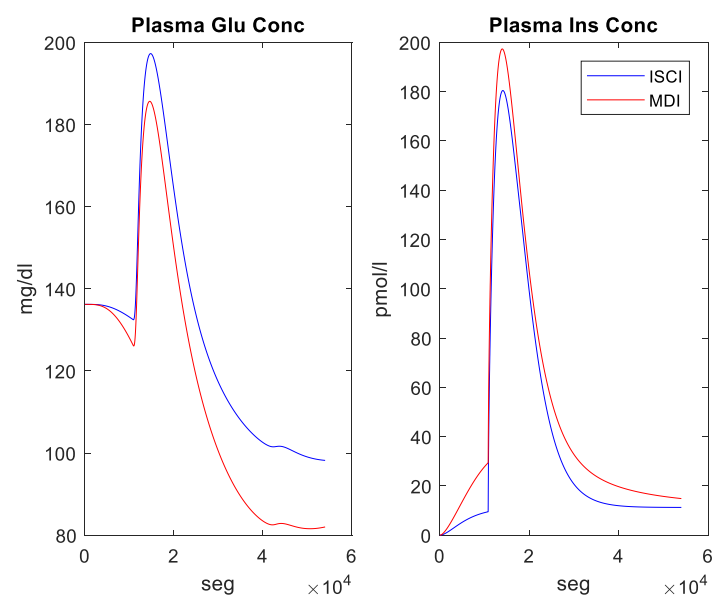

Figura 6: Simulación de infusión de insulina por ISCI vs. MDI.

\subsection{VARIACIÓN DE LA SENSIBILIDAD Y PÉRDIDA DE ABSORCIÓN DE INSULINA}
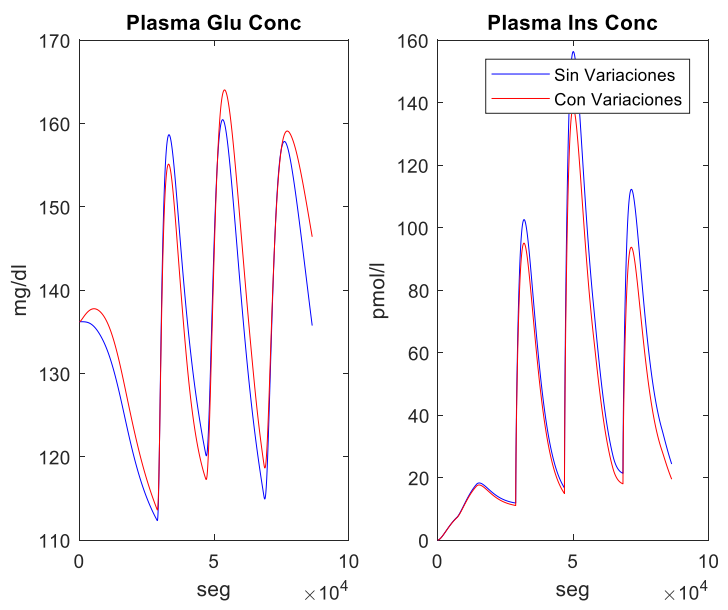

Figura 7: Simulación de las variaciones de varSen y Kinf en un periodo de 24 horas con 3 ingestas de alimento.

En la actualidad no se han publicado modelos matemáticos que representen en totalidad la realidad de un paciente, incluyendo perturbaciones que puedan ser provocadas por factores externos. En esta investigación se proponen los parámetros varSen y
$K_{\text {inf }}$ de las ecuaciones (3) y (7) respectivamente, que pretenden simular perturbaciones controladas de la realidad de un paciente, como las asociadas al uso de bombas de insulina por tiempos prolongados y las variaciones de la sensibilidad del paciente.

En la Figura 7 se puede observar los cambios que generan estas variaciones propuestas simulando pacientes con T1D con tres ingestas de alimento e infusión de insulina en igual proporción.

\section{CONCLUSIONES}

La posibilidad de simular la dinámica glucosa insulina en condiciones reales resulta muy útil para estudiar el comportamiento de la glucosa en un paciente con diabetes; este artículo presentó una propuesta de adaptación de un modelo para un paciente con T1D, el cual permitirá comprender la reacción de la glucosa ante la ingesta adicional de grasas y proteínas, si bien no aportan la misma proporción de azúcar en sangre como los carbohidratos, deben ser tenidos en cuenta para el control del nivel de glucosa.

Con el desarrollo de ésta investigación, se demuestra que desde el campo de la ingeniería se pueden realizar aportes al tratamiento de la diabetes, tales como el desarrollo de herramientas software que permiten la educación de pacientes y profesionales de la salud. Actualmente se desarrollan técnicas de control de glucosa, que si bien no administran insulina en cantidades considerables ayudan a mantener el nivel glucémico durante las noches y entre comidas, simuladores como el presentado en este artículo le aportan a dichas tecnologías la posibilidad de realizar pruebas de control y monitorización en tiempo real sin riesgo; sin embargo, para mejorar el modelo se deben tener en cuenta factores como estrés, actividad física, entre otros.

\section{Agradecimientos}

El presente trabajo se ha realizado dentro del acuerdo de colaboración entre la Universidad Miguel Hernández de Elche y la Universidad del Cauca, Colombia, y en el marco del proyecto DPI201680391-C3-2-R [AEI/FEDER, UE], financiado por la Agencia Estatal de Investigación (AEI) y por la Unión Europea a través del Fondo Europeo de Desarrollo Regional - FEDER - "Una manera de hacer Europa"

\section{English summary}




\section{T1D PATIENT SIMULATOR IN REAL TIME}

\begin{abstract}
This paper develops a real time implementation of a mathematical model that describes the glucose insulin dynamics of a patient with Type 1 Diabetes Mellitus (T1D). In addition, the intake of fats and proteins is contemplated. The model also considers the infusion of exogenous insulin and the variation of insulin sensitivity through the day. Likewise, the engagement of the model to a remote monitoring tool is shown. The real time implementation allows to demonstrate that from engineering is possible to contribute to the T1D insulin treatments.
\end{abstract}

Keywords: Mathematical model, glucose - insulin, T1D, real time.

\section{Referencias}

[1] American Diabetes Association, (2017). Disponible en: http://www.diabetes.org. Último acceso: Junio 2018.

[2] Apablaza, P., Soto, N., Codner, E., (2017). De la bomba de insulin y el monitoreo continuo de glucosa al pancreas artificial. Revista médica de Chile, pp. 630-640.

[3] Berger, M., Rodbard, D., (1989). Computer simulation of plasma insulin and glucose dynamics after subcutaneous insulin injection. Diabetes care, pp. 725-736.

[4] Bergman, R. N., Cobelli, C., (1980). Minimal modeling, partition analysis, and estimation of insulin sensivity. Federation proceedings, vol. 39 , pp. 110.

[5] Chetouani, A., Boutayeb, A., (2006). A critical review of mathematical models and data used in diabetology. Biomedical Engineering Online, pp. 43.

[6] Clemens, A. H., Chang, P. H., Myers, R. W., (1997). The development of Biostator, a Glucose Controlled Insulin Infusion System (GCIIS). Hormone and metabolic research, pp. 23.

[7] Cobelli, C., Renard, E., Kovatchev, B., (2011). Artificial pancreas: past, present, future. Diabetes, pp. 2672-2682.

[8] Dalla Man, C., Raimondo, D. M., Rizza, R. A., Cobelli, C., (2007). GIM, simulation software of meal glucose - insulin model. Journal of Diabetes Science and Technology, pp. 323-330.

[9] Dalla Man, C., Rizza, R. A., Cobelli, C., (2007). Meal simulation model of the glucose - insulin system. IEEE Transactions on biomedical engineering, pp. 1740-1749.

[10] Fabietti, P. G., Canonico, V., Federici, M. O., Benedetti, M. M., Sarti, E., (2006). Control oriented model of insulin and glucose dynamics in type 1 diabetics. Medical and Biological Engineering and Computing, pp. 69-78.

[11] Heroku, (2007). Disponible en: https://www.heroku.com/ Último acceso: Junio 2018.

[12] Kraegen, E. W., Campbell, L. V., Chia, Y. O., Meier, H., Lazarus, L., (1997). Control of blood glucose in diabetics using an artificial pancreas. Internal Medicine Journal, pp. 280-286.

[13] Lehmann, E. D., Deutsch, T., (1992). A physiological model of glucose - insulin interaction in type 1 diabetes mellitus. Journal of biomedical engineering, pp. 235-242.

[14] MongoDB, (2009). Disponible en: https://www.mongodb.com/ Último acceso: Junio 2018.

[15] National Center for Biotechnology Information. PubChem Compound Database, CID: 16132418. Disponible en: https://pubchem.ncbi.nlm.nih.gov/compound/16 132418. Último acceso: Junio 2018.

[16] Nightscout, (2014). Disponible en: https://www.nightscout.info/ Último acceso: Junio 2018.

[17] Pérez, A., Barrios, Y., Monier, A., Berenguer, M., Martínez, I., (2009). Repercusión social de la educación diabetológica en personas con diabetes mellitus. [Artículo en línea] MEDISAN 13(1). Disponible en: http://bvs.sld.cu/revistas/san/vol13_1_09/san11 109.htm. Último acceso: Junio $201 \overline{8}$.

[18] Pérez, C., (2014). Propuesta de algoritmos de predicción de glucosa en pacientes diabéticos. Universidad Politécnica de Madrid, España.

[19] Shichiri, M., Kawamori, R., Yamasaki, Y., Shingeta, Y., Inoue, M., Abe, H., (1977). Computer algorithm of artificial pancreatic beta-cell. Artificial Organs, vol. 1, pp. 141. 
[20] Sociedad Española de Diabetes. (2018). Disponible en: http://www.sediabetes.org. Último acceso: Junio 2018.

[21] Sociedad Española de Diabetes, (2012). Guía de Educación Terapéutica. Disponible en: http://www.diabetes.org. Último acceso: Junio 2018.

\footnotetext{
(c) (9) 2018 by the authors. Submitted for possible open access publication under the terms and conditions of the Creative Commons Attribution CC-BY-NC 3.0 license (https://creativecommons.org/licenses/by-nc/3.0).
} 